\title{
One Dimensional Coupled Thermoelastic Problem Due To Periodic Heating In A Semi-Infinite Rod.
}

\author{
${ }^{1}$ Dr. Ashoke Das, ${ }^{2}$ Dr. Bimalendu Das, \\ ${ }^{1}$ Asst. Prof., Raiganj College(University College), Raiganj, U/Dinajpur.W.B.India. \\ ${ }^{2}$ Retired Professor, North Bengal University, Siliguri, 734430.W.B. India
}

Abstract: This paper is concerned with the determination of the distribution of temperature and displacement in a thin semi-infinite elastic rod when its free end is subjected to periodic heating. It has been pointed out by P.Chadwick(1960) that the rigorous approach, i.c. the approach by way of the coupled equations, to the thermal boundary value problem. In this paper the one dimensional problem of the periodic heating of the free surface of a semi-infinite rod has been solved by a perturbation procedure, approximations upto the first order being retained.

\section{Introduction}

Approach of solving the coupled thermoelastic boundary value problems meets with severe analytical difficulties till 1960, only one partial solution ${ }^{(8)}$ had been published. It has been possible to study the behavior of transient solutions, only for very small or very large values of the tome. In view of this, Lessen .M.(1956) ${ }^{(9)}$ Suggested a procedure to study the approximate solutions of the coupled thermoelastic equations. This one dimensional coupled problem of the periodic heating of the free surface of a semi- infinite rod has been solved by perturbation procedure. Approximations upto the first order being retained.

\section{Method Of Solution.}

Coupled thermoelastic equations in one dimensional case ${ }^{(10)}$ is taken in the form

$$
\begin{aligned}
& \frac{\partial^{2} \theta}{\partial x^{2}}=f \frac{\partial \theta}{\partial t}+g \frac{\partial^{2} u}{\partial x \partial t} \\
& \frac{\partial^{2} u}{\partial x^{2}}-b \frac{\partial \theta}{\delta x}=a \frac{\partial^{2} u}{\partial t^{2}}
\end{aligned}
$$

When $\quad a=\left(\frac{1}{u \tau}\right)^{2}, \quad f=\frac{\rho c l^{2}}{k \tau}, g=\frac{\alpha E l^{2}}{k \tau}, \quad b=\alpha T$

$L$ being the unit of length, $\tau$ the unit of time, $T$ the reference temperature,

$c$ being the unit of temperature, $E$, the unit of stress and $V=\sqrt{\frac{E}{\rho}}, u, t, x, \theta$ have usual meaning.

Let $\theta(x, t)=\theta_{0}(x, t)+b \theta_{1}(x, t)+b^{2} \theta_{2}(x, t)+$

$u(x, t)=u_{0}(x, t)+b u_{1}(x, t)+b^{2} u_{2}(x, t)+$

Making use of (2) into (1) and equating to zero, the different powers of $b$,

We get

$$
\begin{aligned}
\frac{\partial^{2} \theta_{0}}{\partial x^{2}} & =f \frac{\partial \theta_{0}}{\partial t}+g \frac{\partial^{2} u_{0}}{\partial x \partial t} \\
\frac{\partial^{2} u_{0}}{\partial x^{2}} & =a \frac{\partial^{2} u_{0}}{\partial t^{2}} \\
\frac{\partial^{2} \vartheta_{1}}{\partial x^{2}} & =f \frac{\partial \vartheta_{1}}{\partial t}+g \frac{\partial^{2} u_{1}}{\partial x \partial t} \\
\frac{\partial^{2} u_{i}}{\partial x^{2}} & -b \frac{\partial \theta_{i-1}}{\partial x}=\alpha \frac{\partial^{2} u_{i}}{\partial t^{2}}, \quad(i=1,2,3, \ldots \ldots \ldots)
\end{aligned}
$$

The boundary and initial conditions are

$$
\begin{aligned}
& \theta(0, t)=\varphi_{0} \sin n t H(t) \\
& U_{, x}(0, t)=0
\end{aligned}
$$




$$
\begin{aligned}
u(x, 0)=\theta(x .0)= & u_{, t}(x, 0)=0 \\
\mathrm{U}(\mathrm{x}, \mathrm{t}) & \rightarrow 0 \text { as } x \rightarrow \infty \\
\theta(x, t) & \rightarrow 0 \text { as } x \rightarrow \infty
\end{aligned}
$$

These are satisfied if $\theta_{i}(x, t), u_{i}(x, t),(i=0,1,2, \ldots \ldots \ldots \ldots)$ satisfy the following conditions

$$
\begin{aligned}
& \theta_{0}(0, t)=\varphi_{0} \sin n t H(t) \\
& \theta_{i}(0, t)=0 \\
& u_{i, x}(0, t)=u_{i, t}(x, 0)=u_{i,}(x, 0)=\theta_{i}(x, 0)=0 \\
& u_{i}(x, t) \rightarrow 0, \quad \theta_{i}(x, t) \rightarrow 0 \text { as } x \rightarrow \infty(i=1,2,3)
\end{aligned}
$$

The terms

$\theta_{0}(x, t)$ and $u_{0}(x, t)$ in the series (2)form the so-called unperturbed solution of the problem while $\theta_{i}(x, t), u_{i}(x, t)$ give the ith order perturbation solution if $b$ is considered to be the perturbation Parameters. From the table $\mathrm{(11}^{\mathrm{i}}$ values of $\mathrm{b}$ for $\mathrm{Al}, \mathrm{Pb}, \mathrm{Fe}$, and $\mathrm{Cu}$ are seen to be less than 0.01 in each case. This shows that the solution containing terms upto the first power of $b$ will give a fairly accurate approximate results to the exact solution.

\section{Unperturbed Solution.}

The unperturbed solutions $u_{0}(x, t), \theta_{0}(x, t)$ are the solution of

And

$$
\frac{\partial^{2} u_{0}}{\partial x^{2}}=a \frac{\partial^{2} u_{0}}{\partial t^{2}}
$$

$$
\frac{\partial^{2} \theta_{0}}{\partial x^{2}}=f \frac{\partial \theta_{0}}{\partial t}+g \frac{\partial^{2} u_{0}}{\partial x \partial t}
$$

Respectively, Subject to the initial and boundary conditions

$$
\begin{gathered}
u_{0, x}(0, t)=u_{0}(x, 0)=u_{0, t}(x, 0)=\theta_{0}(x, 0)=0 \\
\theta_{0}(0, t)=\varphi \sin n t H(t) \\
u_{0}(x, t) \rightarrow 0, \quad \theta_{0}(x, t) \rightarrow 0, \quad \text { as } x \rightarrow \infty
\end{gathered}
$$

Taking Laplace transforms ${ }^{(12)}$ on these equations and on the boundary conditions give with the help of the other conditions

$$
\begin{gathered}
\frac{d^{2} \bar{u}_{0}}{d x^{2}}=a p^{2} \bar{u}_{0} ; \quad \bar{u}_{0, x}(0, p)=0 \ldots \ldots \ldots \ldots \ldots \ldots \ldots \ldots \ldots \ldots \ldots \ldots \\
\frac{d^{2} \bar{\theta}_{0}}{d x^{2}}=f p \theta_{0}+g p \frac{d \bar{u}_{0}}{d x} ; \quad \theta_{0}(o, p)=\frac{\varphi_{0} n}{n^{2}+p^{2}} \ldots
\end{gathered}
$$

Where $\bar{u}_{0}, \bar{\theta}_{0}$ denote Laplace transform of $u_{0}, \theta_{0}$

The solution of the differential equation (6), subject to the condition that $\ddot{u}_{0}(x, p) \rightarrow 0$ as $x \rightarrow \infty$, is

$$
\bar{u}_{0}=c_{1} e^{-\sqrt{a} p x}
$$

The boundary condition $\bar{u}_{0, x}(0, p)=0$ gives

$c_{1}=0$

Hence $\bar{u}_{0}(x, p)=0$

Taking inverse, $u_{0}(x, t)=0$

Substituting from (9) in the differential equation of (7), the solution of the equation with the boundary condition of (7) is

$$
\bar{\theta}_{0}(0, p)=\frac{n \varphi_{0}}{n^{2}+p^{2}} e^{-\sqrt{ } f p x}
$$

Taking inverse Laplace transform of (11) ${ }^{(3)}$

$$
\theta_{0}(0, x)=\frac{2 \varphi_{0}}{\sqrt{\pi}} \int_{\frac{x}{2} \sqrt{t}}^{\infty} \sin n\left(t-\frac{x^{2} f}{4 \mu^{2}}\right) e^{-\mu} d \mu
$$

\section{First Order Perturbation.}

The first order perturbation is the solution of

$$
\begin{aligned}
& \frac{\partial^{2} u_{1}}{\partial x^{2}}-a \frac{\partial^{2} u_{1}}{\partial t^{2}}=\frac{\partial \theta_{0}}{\partial x} \\
& \frac{\partial^{2} \theta_{1}}{\partial x^{2}}=f \frac{\partial \theta_{1}}{\partial t}+g \frac{\partial^{2} u_{1}}{\partial x \partial t}
\end{aligned}
$$

Subject to the boundary conditions 
$u_{1, x}(0, t)=u_{1, t}(x, 0)=u_{1}(x, 0)=\theta_{1}(x, 0)=0$

$u_{1}(x, t) \rightarrow 0$ as $x \rightarrow \infty$

$\theta_{1}(0, t) \rightarrow 0, \quad \theta_{1}(x, t) \rightarrow 0$, as $x \rightarrow \infty$

Laplace transform of the equation (13) and of the boundary conditions give with the help of the other conditions

$\frac{d^{2} \bar{u}_{1}}{d x^{2}}-a p^{2} \bar{u}_{1}=\frac{d \theta_{0}}{d x} ; \quad \bar{u}_{1, x}(0, p)=0$

Substituting from (11) in the differential equation of (17) and solving, we obtain, on using the condition that $\bar{u}_{1}(x, p) \rightarrow 0$ as $x \rightarrow \infty$,

$$
\bar{u}_{1}=c e^{-\sqrt{a} p x}+\frac{\beta}{a} \frac{e^{-\sqrt{f} p x}}{\sqrt{p}\left(p-\frac{f}{a}\right)\left(p^{2}+n^{2}\right)}
$$

Where $\beta_{0}=n \varphi_{0} \sqrt{f}$

The boundary condition $\quad \ddot{u}_{1, x}(0, p)=0 \quad$ gives

$$
c=\frac{\beta_{0}}{\sqrt{a} f\left(p-\frac{f}{a}\right)\left(p^{2}+n^{2}\right)}
$$

Using the results $(5,9)$, the inverse Laplace transform of $(18)$ is obtained as

$$
\begin{aligned}
& u_{1}(x, t)= \\
& \frac{\beta_{0} H(t-\sqrt{a x})}{\sqrt{a} f\left(n^{2}+\frac{f^{2}}{a^{2}}\right)}\left[e^{\frac{f}{a}(t-\sqrt{a x})}-\frac{f}{a n} \sin n(t-\sqrt{a x})-\cos n(t-\sqrt{a x})\right]- \\
& \frac{\beta_{0}}{a\left(n^{2}+\frac{f^{2}}{a^{2}}\right)}\left[\frac{1}{2} e^{\frac{f t}{a}} \sqrt{\frac{a}{f}}\left\{e^{-\frac{f x}{\sqrt{a}}} \operatorname{erfc}\left(\frac{x}{2} \sqrt{\frac{f}{t}}-\sqrt{\frac{f t}{a}}\right)-e^{\frac{f x}{\sqrt{a}}} \operatorname{erfc}\left(\frac{x}{2} \sqrt{\frac{f}{t}}+\sqrt{\frac{f t}{a}}\right)\right\}-x \sqrt{\frac{f}{\pi}} \int_{\frac{x}{2} \sqrt{\frac{f}{t}}}^{\infty} \cos n(t-\right. \\
& \left.\left.\frac{x^{2} f}{4 \mu^{2}}\right) \frac{e^{-\mu^{2}}}{\mu^{2}} d \mu+\frac{f^{\frac{3}{2}}}{a n \sqrt{\pi}} \int_{\frac{x}{2} \sqrt{\frac{f}{t}}}^{\infty} \sin n\left(t-\frac{x^{2} f}{4 \mu^{2}}\right) \frac{e^{-\mu^{2}}}{\mu^{2}} d \mu\right]
\end{aligned}
$$

On taking Laplace transforms of the equation (14) and of the boundary condition (16), we obtain with the help of (18) and (19)

$$
\frac{d^{2} \bar{\theta}_{1}}{d x^{2}}-f p \bar{\theta}_{1}=\frac{g \beta_{0}}{\sqrt{f}} \frac{p^{2} e^{-\sqrt{a} p x}}{\left(p-\frac{f}{a}\right)\left(p^{2}+n^{2}\right)}-\frac{g \beta_{0} \sqrt{f}}{\sqrt{a}} \frac{p e^{-\sqrt{f} p x}}{\left(p-\frac{f}{a}\right)\left(p^{2}+n^{2}\right)}
$$

And $\quad \bar{\theta}_{1}(0, p)=0, \bar{\theta}_{1}(x, p) \rightarrow 0$ as $x \rightarrow \infty$

The solution of the above equation subject to these boundary conditions is

$$
\bar{\theta}_{1}(x, p)=\frac{g \beta_{0}}{a \sqrt{f}} \frac{p e^{-\sqrt{f} p x}}{\left(p-\frac{f}{a}\right)^{2}\left(p^{2}+n^{2}\right)}-\frac{g \beta_{0} x}{2 \sqrt{a}} \frac{\sqrt{p} e^{-\sqrt{f} p x}}{\left(p-\frac{f}{a}\right)\left(p^{2}+n^{2}\right)}-\frac{g \beta_{0}}{a \sqrt{f}} \frac{p e^{-\sqrt{a} p x}}{\left(p-\frac{f}{a}\right)^{2}\left(p^{2}+n^{2}\right)}
$$

Taking inverse Laplace transform, we obtain from (21),

$$
\begin{aligned}
& \theta_{1}(x, t)=\frac{\beta_{0} g}{2 a \sqrt{f}} \int_{0}^{t} \cos n(t-u) e^{\frac{f u}{a}}\left\{\left(u-\frac{\sqrt{a} x}{2}\right) e^{-\left(\frac{f x}{\sqrt{a}}\right)} \operatorname{erfc}\left[\frac{x}{2} \sqrt{\frac{f}{u}}-\sqrt{\frac{f u}{a}}\right]+(u\right. \\
& \left.\left.+\frac{\sqrt{a x}}{2}\right) e^{\frac{f x}{a}} \operatorname{erfc}\left[\frac{x}{2} \sqrt{\frac{f}{u}}+\sqrt{\frac{f u}{a}}\right]\right\} d u \\
& -\frac{x g \beta_{0}}{4 \sqrt{a}} \int_{0}^{t} \cos n(t-u) e^{\frac{f u}{a}} \sqrt{\frac{a}{f}}\left\{e^{-\left(\frac{f x}{\sqrt{a}}\right)} \operatorname{erfc}\left[\frac{x}{2} \sqrt{\frac{f}{u}}-\sqrt{\frac{f u}{a}}\right]\right. \\
& \left.-e^{\frac{f x}{a}} \operatorname{erfc}\left[\frac{x}{2} \sqrt{\frac{f}{u}}+\sqrt{\frac{f u}{a}}\right]\right\} d u-\frac{\beta_{0} g H(t-\sqrt{a} x)}{a \sqrt{f}} \int_{0}^{t-\sqrt{a x}} \cos n(t-u-\sqrt{a} x) u e^{\frac{f u}{a}} d u
\end{aligned}
$$

Where $\mathrm{H}(\mathrm{t}) \quad=0, \mathrm{t}<0$

$$
=1, \mathrm{t}>0
$$




\section{Discussion.}

It is observed that a factor of the form $e^{\frac{f}{a}(t-\sqrt{a} x)}$ is present in $u_{1}(x, t)$ and in $\theta_{1}(x, t)$. Hence the perturbation solution is valid only so long as $b e^{\frac{f}{a}(t-\sqrt{a} x)}<1$.

This appears to be a characteristic of the one- dimensional problem.

The equations (10) and (12) and (20) and (22), where

$$
\begin{aligned}
& u(x, t)=u_{0}(x, t)+b u_{1}(x, t) \\
& \theta(x, t)=\theta_{0}(x, t)+b \theta_{1}(x, t)
\end{aligned}
$$

Will give the required approximate solution for the displacement and temperature. Also if $g=0$, we obtain the solution of the uncoupled equations of thermoelasticity.

\section{References}

[1] ATKINSON,K.E.(1976); A survey of Numerical Methods for the Solution of Fredholm- Integral Equations of the Second - Kind. Society of Industial and applied Mathematics, Philadelphia,Pa.

CARSLAW,H.S. AND JAEGER, J.C. (1959); Conduction of heat in Solids, $2^{\text {nd }}$ Edn. O.U.P.

SNEDDON, I.N.(1972); The Use of Integral Transform, McGraw Hill, New-York.

SOKOLNIKOFF,I.S.(1956); Mathematical theory of Elasticity, McGraw Hill Book Co. NOWACKI, W.(1986); Thermoelasticity, $2^{\text {nd }}$ End. Pergamon Press.

WASTON,G.N. (1978) ATreatise on the Theory of Bessel Functions, $2^{\text {nd }}$ Edn. C.U.P.

LOVE,A.E.H.(1927) A Treatise on the Mathematical Theory of Elasticity, $4^{\text {th }}$ Edn. Dover Publication.

PARIA.G. (1968): Instantaneous heat sources in an infinite solid, India, J.Mech.Math.(spl. Issue), partI,41.

LESSEN. M.(1968) ; j.Mech.Phys.solids.5,p.

[10] SNEDDON,I.N.(1958); Prog.Roy.Soc.Edin.1959,pp 121-142.

[11] INTERNATIONAL Critical Table of Numerical data.(1927); Vol-II, N.R.C.,U.S.A., McGraw Hill Book Co.

[12] ERDELYI. A.(1954); Tables of Integral Transform, Vol-II, McGraw Hill Book Co. Inc.N.Y. 